\title{
Technology adoption: on the non equivalence of tariffs and quotas*
}

\author{
Berthold Herrendorf ${ }^{\dagger}$
}

Arizona State University

Arilton Teixeira

Fucape Business School

\begin{abstract}
This paper examines the effects of trade policies on the adoption of new technologies. A two-sector model with international-trade is developed where technological progress is neutral. A group of interest (suppliers of skilled labor), acting in coalition, decide which technology will be available for firms. The key findings are the following. (i) With free trade, or a tariff, the best technology is always used; (ii) Under a quota, generally the best technology is not used. In other words, with respect to adoption of new technologies we have equivalence between free trade and tariffs. A quota generates resistance to new technologies while with free trade or tariffs this resistance is eliminated.
\end{abstract}

Keywords: coalitions; technology adoption; quota; tariff.

Received in 05/05/2007; revised in 10/20/2007; accept in 10/29/2007.

\section{Corresponding authors:}

$\dagger$ †.P. Carey School of Business, Department of

Economics Arizona State University, Tempe, AZ 85287-3806, USA.

e-mail: berthold.herrendorf@asu.edu
\$ Associate Professor at the Fundacao Capixaba de Pesquisa, Av Fernando Ferrari, 1358 Vitoria, ES 29075-010 Brazil. e-mail: arilton@fucape.br

* We are grateful to Ed Prescott and Jim Schmitz. I also thank Juan Carlos Conesa, Carlos Díaz, Ron Edwards, Thomas Holmes and Timothy Kehoe.

Editor's note: This paper was accepted by Alexsandro Broedel Lopes. 


\section{INTRODUCTION}

There is evidence that total factor productivity (TFP henceforth) differs across countries. ${ }^{1}$ It has been argued that part of this difference is due to the resistance to the adoption of new technologies. That is, interest groups (coalitions) in the domestic economy would stop the adoption of new technologies. Empirical evidence of this resistance has been documented by Mokyr [11,12,13] and Olson [15].

In this paper we study how trade policy (free trade, tariffs and quotas) affects resistance to new technologies. We construct a two sector model with exogenous technological progress and international trade. The economy is small compared to the international market (that is, the domestic economy takes the international prices as given). One sector uses only unskilled labor. The other sector uses unskilled and skilled labor. The skilled workers are the only ones able to use new technologies in this sector. Since they are the only ones able to use the new technologies, we assume that the skilled workers as a coalition decide which technology the firms will be allowed to use. The main results are the following: (i) Under free trade or tariffs there is no blocking of new technologies; (ii) under quota there will be resistance to the adoption of new technologies.

This paper follows the research line of Holmes and Schmitz [9] and Parente and Prescott $[16,17]$. In these papers, groups or coalitions can affect the relative prices through resistance to new technologies, thereby transferring income from the rest of the society to the coalition members. In Parente and Prescott [16,17], in a closed economy, the labor unions have the power (they called it monopoly rights) to decide which technology will be used. In Holmes and Schmitz [9] workers have the power to resist to new technologies. The key result is that they do not exercise their power if the economy operats in a free trade environment. In contrast, in a closed economy (quota equal to zero), the coalition exercise its power and it blocks new technologies.

Our paper is closely related to Bhagwati [3,4] and Holmes and Schmitz [9]. We extended Holmes and Schmitz [9] since we analyze not only free trade and a closed economy (zero quota), but also the effects of different sizes of quotas and different sizes of tariffs. We conclude that with respect to technology adoption tariffs and quotas are not equivalent.

Bhagwati $[3,4]$ has pointed out that there is an equivalence between tariffs and quotas as long as we have perfect competition among producers (domestic and foreign) and among those with the right to import. That is, for each price and volume of imports generated by a tariff there exists a quota that generates the same price and volume of imports. Our results indicated that with respect to adoption of new technologies the equivalence is between free trade and tariffs since both eliminate resistance. ${ }^{2}$

One result relating trade policy and resistance to new technologies is in line with the empirical evidence relating trade liberalization and increment in total factor productivity. By trade liberalization we mean the elimination of non tariff barriers (quotas) as well as reduction of the variance of the tariffs across sectors (as well as reduction of the size of tariffs). According to our model, if the introduction of a quota generates resistance to use new and more productive technologies the elimination of a quota should come with a short run increase in the productivity because new technologies were not in use because resistance are now adopted. Ferreira and Rossi [6] and Muendler [14] studying Brazil, Pavcnik [18] and Tybout,

\footnotetext{
${ }^{1}$ See Dollar and Wolf [5], Harrigan [7,8] and Prescott [19].

${ }^{2}$ We should stress that Bhagwati $[3,4]$ also pointed out that once perfect competition is eliminated in one of this markets this equivalence no longer exists. This result does not apply to our case since since we keep the assumption of perfect competitive in all of these markets.
} 
Mello and Corbo [20] studying Chile and Kim [10] sudying Korea found a strong correlation between short run growth in productivity and trade liberalization. Even though they generally do not differentiate impacts of quota and tariffs, Muendler [14] tested the difference for the Brazilian case. More specifically, he estimates the effects of a reduciotn of $1 \%$ on the level of protection (a reduction of 1\% in the tariff level or an increment of 1\% in the market share of imports). Using Brazilian data from 1986-1998 he estimated the effects of a quota on the TFP as being ten times bigger than the effect of a tariff. ${ }^{3}$

Finally we can say that our economy with free trade or tariff is equivalent to Parente and Prescott $[16,17]$ economy without monopoly rights. In their model, if no group has the power to block new technologies (as they say, there are no monopoly rights), the firms will always use the most productive technology. In our case the economy with free trade or tariff has similar results toward adoption of new technologies. Competition with foreign firms is a sufficient condition to stop interest groups exercising their monopoly rights. This result goes in the same direction as the evidences found by Baily [1] and Baily and Gersbach [2].

This paper is divided in four sections including this introduction. In the second section we develop the model. The third section analyzes the three trade policies. The fourth section compare the results and concludes.

\section{THE MODEL ECONOMY}

There are two periods indexed by $t \in\{1,2\}$ and there is no uncertainty. There are five goods in each period: the consumption goods $y_{t}$ and $z_{t}$, time allocated to the production of $y_{t}, l_{y t}$, and two types of time $l_{i t}, i \in\{1,2\}$, allocated to the production of $z_{t}$. The period commodity space is $X_{t} \equiv \square^{5}$ and a point in $X_{t}$ is $x_{t}=\left(y_{t}, z_{t}, l_{y t}, l_{1 t}, l_{2 t}\right)$. By assumption we have a small and open economy in the sense that it takes the world market prices of the goods $y$ and $z$ as given. Labor is assumed to be immobile across countries. Good $y_{t}$ is the numeraire and its price is set equal to one. The other prices are $\left\{p_{y t}, p_{z t}, p_{z t}^{*}, w_{y t}, w_{1 t}, w_{2 t}\right\}$, where variables without and with a superscript $*$ are home market prices and world market prices, respectively.

There is a finite measure of agents. All agents have the same preferences over $X_{1} \times X_{2}$ represented by $\sum_{t=1}^{2} \beta^{t-1}\left(y_{t}^{\alpha} z_{t}^{1-\alpha}\right)^{\rho} / \rho$, where $\beta \in(0,1)$ is the discount factor and $1>\rho$ is the parameter that defines the coefficient of relative risk aversion. The agents differ with respect to the endowment of labor. There are two types: a measure $\lambda_{i}>0, i \in\{1,2\}$ of type- $i$ agents endowed with one unit of type $i$ time per period. ${ }^{4}$ There is no capital and also no borrowing or lending. Therefore, consumers' and firms' problems are static (we will omit the time arguments from now on as long as this omission does not cause confusion). In order to describe the consumers' problems, we define the period consumption sets of type $i \in\{1,2\}$ :

$$
X_{i} \equiv\left\{x \in X_{+}: l_{y}+l_{i} \leq 1, l_{j}=0 \text { for } j \neq i\right\}
$$

\footnotetext{
${ }^{3}$ See Muendler [14, page 32].

${ }^{4}$ The specification of the two technologies below will imply that one can think of type- 1 agents (type- 2 agents) as being unskilled (skilled).

BBR, Braz. Bus. Rev. (Eng. ed., Online),

Vitória, v.4, n. 3, Art. 3, p. 195-217, sep.-dec. 2007

www.bbronline.com.br
} 
We can see in (1) that there is no difference between the time that either type allocates to the production of good $y$. We justified this later on when we specify the technologies. The period problem of an agent of type $i$ is

$$
\max _{x \in X_{i}} u(x) \text { s.t. } y+p_{z} z \leq w_{y} l_{y}+w_{i} l_{i} .
$$

That is, a type $i$ chooses from his period consumption set the quantities of the two consumption goods and the time allocated to the two sectors so as to maximize his period utility, respecting his period budget constraint.

Now let's specify the production side of the economy. We have three sectors. Firms in each sector operate a constant-returns-to-scale technology. Since there is perfect competition firms make zero profits. The technology of each sector depends on the state variables represented by $s=(a, b, t)$ where $a$ and $b$ are integers with $a<b \leq t$. In other words, the state space is $S \equiv\left\{(a, b, t) \in Z^{2} \times\{1,2\}: a<b \leq t\right\}$. We specify below how $a$ and $b$ are determined.

The first sector produces the good $y$. Given the state variables in period $t s=(a, b, t)$, the production set of a representative firm is

$$
X_{3}(s)=\left\{x \in X_{+}: y \leq \pi^{t} l_{y}, z=0\right\},
$$

where $\pi \in(1, \infty)$, implying that in sector 1 there is exogenous technological progress. As stated before, there is no difference in productivity between type 1 and type 2 time when they work in the first sector. This justifies the previous assumption that the time of either type is the same good when it is allocated to the first sector. The second sector produces the good $z$. Given $s=(a, b, t)$ in period $t$, the production set of the representative firm is

$$
X_{4}(s)=\left\{x \in X_{+}: z \leq \gamma^{a} l_{1}+\gamma^{b} l_{2}, y=0\right\},
$$

where $\gamma \in(\pi, \infty)$ and $a$ and $b$ are non-negative integers. Since $a<b$ the time of type 2 (the skilled type) is more productive in sector 2 than time of type 1 (the unskilled type). The third sector is the international trade sector. We model the international trade as a production function by which each of the two consumption goods can be transformed into the other one. Given $s=(a, b, t)$ in period $t$, the production set of a representative firm is

$$
X_{5}(s)=\left\{x \in X: y+p_{z}^{*} z=0, l_{y}, l_{1}, l_{2} \geq 0\right\} .
$$

Equation (3c) implicit shows that there are neither transportation cost nor borrowing or lending.

Given the above structure of our economy the firm's problem in each sector is static. Their choices in period 1 have no effects on the problems in period 2 . Specifically, in each period, given the realization of $s$, the representative firm of sector $j, j \in\{1,2,3\}$, maximize profits:

$$
\max _{x \in X_{j+2}(s)} p_{y} y+p_{z} z-w_{y} l_{y}-w_{1} l_{1}-w_{2} l_{2} .
$$

Finally, we turn to the law of motion of the state variables. We assume that the type- 2 agents acting as a group (coalition) have the power to choose $b .^{5}$ Specifically, given $s=(a, b, t-1)$ at the beginning of period $t$ type 2 agents choose $b^{\prime}$, the technology that firms

${ }^{5}$ We address the question if it is optimal for a skilled agent to stay in the coalition later on. 
will be allowed to use in period $t$. The set of choices is $b^{\prime} \in\{b, \ldots, t\}$. Given the choice of $b^{\prime}$, $a^{\prime}$ is determined as follows: if $b^{\prime}>b$, then $a^{\prime}=b$; if $b^{\prime}=b$, then $a^{\prime}=a$. In words, if the type2 agents choose a better technology for the period $t$, then type- 1 agents gain access to the technology that the type- 2 agents were previously using. If the type- 2 agents choose to continue with the technology they were previously using, then the type- 1 agents too will continue with the technology they were previously using. Note that at the beginning of period 1 , there is no old technology. We set the initial values $s=(-1,0,0)$.

The coalition's problems of choosing the optimal technology in the two periods are described next. Given that the state at the beginning of period 1 is $s=(-1,0,0)$, the coalition's problem is:

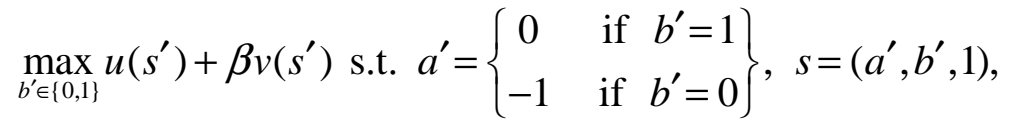

where $\mathrm{u}\left(\mathrm{s}^{\prime}\right)$ and $\mathrm{v}\left(\mathrm{s}^{\prime}\right)$ denote the period utility and the continuation utility of an agent of type 2 for state $s^{\prime}$, respectively. ${ }^{6}$ Given a realization of the state $s=(a, b, 1) \Leftarrow S$ at the beginning of period 2, the coalition's problem is:

$$
\max _{\mathrm{b}^{\prime} \in\{\mathrm{b}, . ., 2\}} \mathrm{u}\left(\mathrm{s}^{\prime}\right) \text { s.t. } \mathrm{a}^{\prime}=\left\{\begin{array}{cc}
b ; & b^{\prime}>b \\
a ; & b^{\prime}=b
\end{array}\right\}, \mathrm{s}^{\prime}=\left(\mathrm{a}^{\prime}, \mathrm{b}^{\prime}, 2\right) .
$$

We are now able to define an equilibrium for our economy. This equilibrium is divided in two parts: a static and a dynamic part. We call the former the period competitive equilibrium and the latter the recursive equilibrium. The reason or this distinction is the following. Since there is neither borrowing or lending nor capital accumulation, the choices of firms and the choices of the consumers in period 1 have no effect on the problem in period 2. That is, the coices of the consumers are static given the choice of technology.

Definition 1 (Period Competitive Equilibrium) Given a realization of $s \in S$, a period competitive equilibrium is a set of prices $\hat{p}(s)=\left\{\hat{p}_{y}(s), \hat{p} z=, \hat{w}_{y}(s), \hat{w}_{1}(s), \hat{w}_{2}(s)\right\}$, allocations $\left\{\hat{\mathfrak{x}}_{i}(s)\right\}_{i=1}^{5}$, and imports ${ }^{7}\left\{\hat{y}^{*}(s), \hat{z}^{*}(s)\right\}$ such that:

(i) $\hat{x}_{i}(s), i \in\{1,2\}$, solves the period problem of the representative agent of type $i$;

(ii) $\hat{x}_{j}(s), j \in\{1,2,3\}$, solves the period problem of the representative firm of type $j$;

(iii)markets clear, that is, $\lambda_{1} \hat{x}_{1}(s)+\lambda_{2} \hat{x}_{2}(s)=\hat{x}_{3}(s)+\hat{x}_{4}(s)+\hat{x}_{5}(s)$.

Definition 2 (Recursive Equilibrium) A recursive equilibrium is a set of price functions $\hat{p}$, allocation functions $\left\{\hat{x}_{i}\right\}_{i=1}^{5}$, import functions $\left\{\hat{y}^{*}, \hat{z}\right\}$, and technology choice functions $\left\{b_{t}\right\}_{t=1}^{2}$ such that:

(i) $\forall s \in S \hat{\hat{p}}(s),\left\{\hat{x}_{i}(s)\right\}_{i=1}^{5},\left\{\hat{y}^{*}(s), \hat{z}^{*}(s)\right\}$ is a period competitive equilibrium;

(ii) $b_{1}(-1,0,0)$ solves $(5 \mathrm{a})$ and $b_{2}(s)$ solves $(5 \mathrm{~b}) \forall s=(a, b, 1) \in S$.

For future use we define what we mean by blocking the use of efficient technologies.

\footnotetext{
${ }^{6}$ As will become clear in the proof of Proposition 1 below, $\mathrm{u}\left(\mathrm{s}^{\prime}\right)$ and $\mathrm{v}\left(\mathrm{s}^{\prime}\right)$ are unique for the present model.

${ }^{7}$ Note that negative imports are exports, so we can talk about the imports of both goods.
} 
Definition 3 [Blocking of Technologies] We say that there is blocking of new technologies if and only if there is a period $t, t \in\{1,2\}$, in which $b<t$.

\section{TRADE POLICY AND TECHNOLOGY ADOPTION}

\subsection{Free Trade}

We start studying the free trade economy. Since we are assuming a small open economy the relative prices of the two consumption goods in the home market are determined by their values in the world market, exogenously given to our economy: $\quad p_{y}=p_{y}{ }^{*}=1$ and $p_{z}$ $=p_{z}{ }^{*}$. We choose the following specification: ${ }^{8}$

$$
p_{z t}^{*}=\eta \frac{\pi^{t}}{\gamma^{t}}, \quad \eta \in(1, \gamma)
$$

We first characterize the period-equilibrium for a given realization of the state $s \in S$ and then the recursive equilibrium. The derivation of the period equilibrium involves four steps. First, from the consumer problems we get the demand functions of goods y and $z$. Given the consumer's utility function it is straightforward to show that they spend the shares $\alpha$ and 1 - $\alpha$ of their incomes on a period consuming goods $y$ and $z$. Second, from the firm's problem, (3), we get the wages that are equal to the marginal products. Third, using the results obtained in step one and two we find two cases: if $b=t$, then the type- 1 agents (type- 2 agents) work only in sector 1 (sector 2 ); if $b<t$, then both types work only in sector $1 .{ }^{9}$ Fourth, to find the imports and exports we just substitute the consumer demands and the home production of the two goods. The results are summarized by the following lemma. The proof and the detailed characterization of the different equilibria can be found in the appendix.

Lemma 1(Period-Equilibrium with Free Trade) Suppose that there is free trade, that $p_{z}{ }^{*}$ is given by (6), and that $s \in S$ is the given state. Then, there are two cases.

(i) If $s=(a, t, t) \in S$, then there is a unique period equilibrium. In this equilibrium type-1 agents work in sector 1 and type-2 agents work in sector 2.

(ii) If $s=(a, b, t) \in S$ with $b<t$, then there is a unique period equilibrium. In this equilibrium both types work in sector 1 .

Below we will analyze tariffs and quotas on $z$, so we are interested in knowing under which conditions $z$ is imported under free trade. The next lemma makes these conditions explicit. Its proof follows directly from the expressions for the imports of $z$ under free trade, which can be found in the proof of Lemma 1 in the appendix.

\footnotetext{
${ }^{8}$ This specification implies that under free trade type- 1 agents and type- 2 agents work in sector 1 and in sector 2 , respectivelly (Lemma 1 below shows the details). Although starting with this case makes the algebraic manipulations easier, the results do not change if we abandoned this specification.

${ }^{9}$ In particular, $\mathrm{p}_{\mathrm{z}}{ }^{*}>\pi^{\mathrm{t}} \gamma^{\mathrm{b}-\mathrm{t}}$ *turns out to be the condition for the equilibrium to be such that the type-2 agents work only in sector 2 . Given $p_{z t}=\eta \pi^{*} \gamma^{-}$, this condition is equivalent to $b=t$ and $\eta>1$; see Lemma 1 . Moreover, $p_{z t}<$ $\pi^{\mathrm{t}} \gamma^{\mathrm{a}-\mathrm{t}}$ turns out to the condition for the equilibrium to be such that the type-1 agents work only in the sector 1 . Given $\mathrm{p}_{\mathrm{zt}}{ }^{*}=\eta \pi^{\mathrm{t}} \gamma^{-\mathrm{t}}$, this condition is equivalent to $\mathrm{a}<\mathrm{t}$ and $\eta<\gamma$; see Lemma 1 . So the assumption that $\eta \in(1, \gamma)$ is crucial. 
Lemma 2(Period-Equilibria With Positive Imports of $z$ ) Suppose that there is free trade, that $p_{z}{ }^{*}$ is given by (6), and that $s \in S$ is the given state. Then $z$ is imported in the unique period equilibrium if and only if either $b<t$ or $b=t$ and

$$
\lambda_{2} \alpha \eta<\lambda_{1}(1-\alpha) \text {. }
$$

The interpretation of this result is as follows. If $b<t$, then $z$ is not produced at all and so must be imported. If $b=t$, then the unskilled agents produce $y$ and the skilled agents produce $z$. Given this, $z$ is the more likely to be imported the smaller is the mass of the skilled agents relative to that of the unskilled agents, the larger is $z$ 's expenditure share $1-\alpha$ relative to that of $y$, and the smaller is $z$ 's relative price in terms of $y$ (i.e. the smaller is $\eta$ ).

The next step is to determine the optimal choice of the technology under free trade for the skilled workers. To understand this decision we should notice that in the free trade enviroment the small open economy takes the relative prices as given by the world market, so the coalition's problem of choosing the technology is equivalent to choosing the technology that maximizes a type 2 period income. But a type 2 has income equal to $p_{z}$ times the marginal product of type 2's time used to produce $z$. Given the relative prices a type 2's income is maximized by maximizing the marginal product of type- 2 labor. That is, choosing the best technology is strictly preferred. Therefore new technologies are never blocked and technological progress and growth in sector 2 are as large as possible. This result is state in the next proposition. The formal proof can be found in the appendix.

Proposition 1 (Recursive Equilibrium with Free Trade) Suppose that there is free trade and that $p_{z}{ }^{*}$ is given by (6). Then there is a unique recursive equilibrium. This equilibrium has the following properties: (a) Type-1 agents work in sector 1 and type-2 agents work in sector 2. (b) The most productive technology is used in both periods.

\subsection{Tariffs}

In this section we study the consequences of a tariff $\tau>0$ on the imports of good $z$. The presence of a tariff introduces a wedge between the domestic relative price of $z_{t}$ and the world market price. Using (6) the relative price of $z$ in the home market is given by: ${ }^{10}$

$$
p_{z t}=(1+\tau) p_{z t}^{*}=(1+\tau) \eta \frac{\pi^{t}}{\gamma^{t}}, \quad \eta \in(1, \gamma)
$$

It is natural to consider only parameter values for which the home country imported $z$ under free trade, so we assume that either $b<t$ or $b=t$ and (7) holds.

The introduction of a taiff makes it necessary to change the definition of equilibrium. First, after the introduction of a tariff $p_{z}$ is now given by (8). Second, the resulting revenue from the tariff needs to be taken care off. We assume that there is a government that collects it and throws it into the ocean. With this assumption the introduction of a tariff does not have any other effect on the definition equilibrium. This simplifies the proofs although it is not crucial for our results.

To characterize the equilibrium we will follow the same steps given in the free trade case. As in the free trade we start with the period equilibrium. We should stress a change that

\footnotetext{
${ }^{10}$ We only consider constant tariffs, so a BGP exist.
} 
can happened once we introduce a tariff. For sufficiently high tariff values the relative price of $z$ can become so high that additional agents start working in sector 2 and the economy switches to exporting good $z$. We exclude this case because it contradicts our assumption that $z$ is imported. Moreover, it is a trivial result the skilled will no longer have incentives to block new technologies when the good that they produced is exported and so they stand in direct competition with the other producers. We summarize in the next lemma the results for the period equilibrium when $z$ is imported (the proof and the details of the equilibria can be seen in the appendix).

Lemma 3(Period-Equilibrium with Tariff) Suppose that there is a constant tariff on the imports of $z$, that $p_{z}{ }^{*}$ is given by (6), that (7) holds, and that $s \in S$ is the given state. Then there are five cases.

(i) If the tariff satisfies

$$
\tau<\frac{\gamma^{t-b}}{\eta}-1
$$

then there is a unique period equilibrium where $z$ is imported and both types work in the sector 1 .

(ii) If the tariff satisfies

$$
\tau=\frac{\gamma^{t-b}}{\eta}-1
$$

then there is a continuum of period equilibria where $z$ is imported and type 1 works in sector 1 . The allocation of working time of type 2 is indeterminate. ${ }^{11}$

(iii)If the tariff satisfies

$$
\frac{\gamma^{t-b}}{\eta}-1<\tau<\min \left\{\frac{\gamma^{t-a}}{\eta}-1, \frac{\lambda_{1}(1-\alpha) \gamma^{t-b}}{\lambda_{2} \alpha \eta}-1\right\},
$$

then there is a unique period equilibrium where $z$ is imported, type 1 works in sector 1 and type 2 works in sector 2.

(iv) If the tariff satisfies

$$
\tau=\frac{\gamma^{t-a}}{\eta}-1<\frac{\lambda_{1}(1-\alpha) \gamma^{t-b}}{\lambda_{2} \alpha \eta}-1,
$$

then there is a continuum of period equilibria where $z$ is imported and type 2 works in sector 2. The allocation of working time of type 1 is indeterminate.

(v) If the tariff satisfies

${ }^{11}$ That is type 2's working time allocation can take on a continuum of values. 


$$
\tau>\frac{\gamma^{t-a}}{\eta}-1 \quad \text { or } \quad \tau \geq \frac{\lambda_{1}(1-\alpha) \gamma^{t-b}}{\lambda_{2} \alpha \eta}-1
$$

then in any period equilibrium $z$ is imported.

The intuition for Lemma 3 is similar to that for Lemma 1. As can be seen from (8), the relative price of $z$ is proportional to the tariff. So if the tariff is relatively low, then $p_{z t}$ too is relatively low. Good $z$ is then not produced in the home country and its entire consumption must be imported. If the tariff is in the middle range, then $p_{z t}$ and the production of $z$ in the home country too are in the middle range. Good $z$ is then imported. If the tariff is relatively high, then $p_{z t}$ and the production of $z$ in the home country too are relatively high. Good $z$ is then exported.

We now characterize the optimal choice of technology under a tariff. As it had happened with free trade, under a tariff there will be no blocking. That is, free trade and a tariff are equivalent with respect to the adoption of new technolgies. The intution for this result is also similar to the one given in the free trade case. A tariff introduces a wedge between the domestic price and the international market price. But, the relative price in the home market is still given to the coalition. The coalition cannot manipulate this distorted relative price in its favor. Therefore, to maximize the income (and the utility) of its member the coalition has to choose the highest feasible $b$. As a result, new technologies are never blocked and technological progress is as large as possible (as we will see below, this is different under a quota). The next proposition summarized this results (the proof is in the appendix).

Proposition 2 (Recursive Equilibrium with Tariff) Suppose there is a constant tariff on the imports of $z$, that $p_{z}{ }^{*}$ is given by (6), and that (7) holds. Then there are three cases.

(i) If the tariff satisfies

$$
\tau<\frac{\lambda_{1}(1-\alpha)}{\lambda_{2} \alpha \eta}-1 \leq \frac{\gamma}{\eta}-1 \quad \text { or } \quad \tau<\frac{\gamma}{\eta}-1<\frac{\lambda_{1}(1-\alpha)}{\lambda_{2} \alpha \eta}-1
$$

then there is a unique recursive equilibrium. In this equilibrium: (i) $z$ is imported. (ii) Type-1 works in sector 1 and type-2 works in sector 2. (iii) The most productive technology is used in both periods.

(ii) If the tariff satisfies

$$
\tau=\frac{\gamma}{\eta}-1<\frac{\lambda_{1}(1-\alpha)}{\lambda_{2} \alpha \eta}-1
$$

then there is a continuum of recursive equilibrium. In these equilibrium (i) $z$ isalways imported. (ii) Type-2 works in sector 2 and the allocation of working time of type 1 is indeterminate. (iii) The most productive technology is used in both periods. 


$$
\tau<\frac{\lambda_{1}(1-\alpha)}{\lambda_{2} \alpha \eta}-1 \leq \frac{\gamma}{\eta}-1 \quad \text { or } \quad \tau<\frac{\gamma}{\eta}-1<\frac{\lambda_{1}(1-\alpha)}{\lambda_{2} \alpha \eta}-1
$$

then in any recursive equilibrium $\mathrm{z}$ is not imported.

\subsection{Quotas}

In this part of the paper we analyze our model economy once we introduce a quota on the imports of good $z$. We introduce a quota in the following way. In each period the total amount of good $z$ that is allowed to be imported is assumed proportional to the amount produced domestically. That is, in period $t$ the economy imports a constant fraction $Q$ of the total production of $z_{t}$

$$
z_{t}^{*}=Q z_{t}
$$

where $Q \in[0, \infty)$. Obviously we again need to require that $z$ be imported under free trade, so we again assume that $b<t$ or $b=t$ and (7) holds. The quota has to satisfy two constraints. First, it has to bind. That is, the amount imported with a quota has to be smaller than the amount imported under free trade. Second, the quota has to increase the domestic relative price of $z$ above the world market price.

Once we introduce a quota, we have to adapt our definition of equilibrium. First, since we want the quota to be binding, the imports of $z$ must be equal to the quota given by (15) . Second, the introduction of a quota generates income to those that import good $z$. This income comes from the difference of domestic and international prices. We will keep our assumption that the government collects this income and throws the tax revenues away. Once more, this assumption leaves the market clearing conditions unaffected. Although simplifying our proofs our results do not depend on this assumptions.

As before we now characterize the equilibrium under a quota. We start with the period equilibrium. We should pay attention to two points. First, the quota breaks the link between the relative price of $z$ in the home market and in the world market. In contrast to the previous two cases, $p_{z}$ is now determined by the equilibrium conditions of the domestic economy. Second, a very restrictive quota on $z$ may increase $p_{z}$ by so much that the economy switches to exporting $z$. Again we exclude such cases. The valid cases are summarized by the following lemma. The proof and the details of the equilibria can again be found in the appendix.

Lemma 4(Period-Equilibrium with Quota) Suppose that there is a quota $Q$ on the imports of good $z$ as in (15), that $p_{z}{ }^{*}$ is given by (6), that (7) holds, and that the state $s \xi S$ is given. Then, there are four cases.

(i) If the quota satisfies

$$
Q<\frac{\lambda_{1}(1-\alpha)}{\lambda_{2} \gamma^{b-a}}-\alpha
$$

then there is a unique period equilibrium where: (i) the quota binds and $p_{z t}>p_{z t}{ }^{*}$. (ii) type-1 agents work in both sectors and type-2 agents work in sector 2. 


$$
\frac{\lambda_{1}(1-\alpha)}{\lambda_{2} \gamma^{b-a}}-\alpha \leq Q \leq \frac{\lambda_{1}(1-\alpha)}{\lambda_{2}}-\alpha \quad \text { and } \quad Q<\frac{\lambda_{1}(1-\alpha) \gamma^{t-b}}{\lambda_{2} \eta}-\alpha
$$

then there is a unique period equilibrium where: (i) the quota is binding and $p_{z t}>$ $p_{z t}{ }^{*}$. (ii) type-1 agents work in sector 1 and type-2 agents work in sector 2.

(iii)If the quota satisfies

$$
Q>\frac{\lambda_{1}(1-\alpha)}{\lambda_{2}}-\alpha
$$

and $b<t$ then there is a unique period equilibrium where: (i) the quota is binding and $p_{z t}>p_{z t}{ }^{*}$. (ii) type-1 agents work in sector 1 and type-2 agents work in both sectors.

(iv)If none of the conditions (16a)-(16c) are satisfied, then in any period equilibrium the quota is not binding and $p_{z t}>p_{z t}{ }^{*}$.

Now let's give some intuition for the above lemma. If the quota is relatively large, then the relative price of $z$ is so low that no equilibrium exists in which imports under the quota fall short of imports under free trade. If the efficient technology is not used, then both types work in the first sector under free trade. Under a relative large quota, the relative price of $z$ can increase sufficiently relative to free trade that sufficiently many agents of type 2 start to work in the second sector to make up for the reduction in imports. If the efficient technology is used, then there are two case. On the one hand, the binding quota can be in a middle range where the relative price of $z$ is in a middle range too so that type- 1 agents work in the first sector and type- 2 agents work in both sectors. On the other hand, the binding quota can be rather tight so that the relative price of $z$ becomes so high that some type-1 agents start to work in the second sector to make up for the small quantity of imports.

Now let us find the optimal choice of technology under the quota for the coalition members. The main difference with respect to free trade and tariff is the following. Once we introduce a quota, the domestic relative prices are no longer given. That is, the domestic relative price of $z$ is determined by the market clear conditions of our home economy. Once the domestic price of good $z$ is endogenous (and dependent on the technology choice) the coalition's problem of maximizing the utility of its representative member is no longer equivalent to maximizing the marginal product of type-2 labor. Now, the coalition's choice of technology affects both the marginal product of a type 2 labor and the relative price of $z$. By blocking a newer technology the coalition increases the relative price of z. Under certain conditions this increase will be sufficient to compensate the smaller marginal productivity of a type 2 labor, increasing the income the utility of the coalition members. Therefore, under certain conditions we will have blocking of the newest technology. This will generate the major difference between the free trade, the tariff and the quota economy. The equivalence of tariffs and quotas is no longer true. The next proposition formalizes this argument. (the proof is the appendix).

Proposition 3 (Recursive Equilibrium with Quota) Suppose that there is a quota $Q$ on the imports of good $z$ as in (15), that $p_{z}{ }^{*}$ is given by (6), and that (7) holds. Then, there are four cases.

(i) Supose the quota satisfies 


$$
Q<\frac{\lambda_{1}(1-\alpha)}{\lambda_{2} \gamma^{2}}-\alpha
$$

then if and only if

$$
\gamma^{(1-\alpha) \rho}-1<\beta \pi^{\alpha \rho} \gamma^{2(1-\alpha) \rho}\left[\gamma^{\alpha \rho}-1\right]
$$

there is a unique recursive equilibrium where: (i) the quota binds and $p_{z t}>p_{z t}{ }^{*}$. (ii) Type-1 agents work in both sectors and type-2 agents work in the second secto. (iii) The most productive technology is blocked in the first period and used in the second period. Otherwise the most productive technology is used in both periods.

(ii) If the quota satisfies

$$
\frac{\lambda_{1}(1-\alpha)}{\lambda_{2} \gamma^{2}}-\alpha \leq Q<\frac{\lambda_{1}(1-\alpha)}{\lambda_{2} \gamma}-\alpha
$$

then if and only if

$$
\gamma^{(1-\alpha) \rho}-1<\beta \pi^{\alpha \rho} \gamma^{2(1-\alpha) \rho}\left\{\left[\frac{\lambda_{1}(1-\alpha)}{\lambda_{2}(\alpha+Q) \gamma}\right]^{\alpha \rho}-1\right\}
$$

there is a unique recursive equilibrium where: (i) the quota is binding and $p_{z t}>$ $p_{z t}{ }^{*}$ in all periods. (ii) Type-1 agents work in the first sector and type-2 agents work in the second sector. (iii) the most productive technology is blocked in the first period and used in the second period. Otherwise the most productive technology is used in both periods.

(iii)If the quota satisfies

$$
\frac{\lambda_{1}(1-\alpha)}{\lambda_{2} \gamma}-\alpha \leq Q<\frac{\lambda_{1}(1-\alpha)}{\lambda_{2} \eta}-\alpha,
$$

then there is a unique recursive equilibrium where: (i) the quota is binding and $p_{z t}$ $>p_{z t}{ }^{*}$. (ii) type-1 agents work in sector 1 and type-2 agents work in sector 2. (iii) the most productive technology is used in both periods.

(iv) If the quota satisfies

$$
Q \geq \frac{\lambda_{1}(1-\alpha)}{\lambda_{2} \eta}-\alpha
$$
$p_{z t}{ }^{*}$.

then there no is recursive equilibrium such that the quota is binding and $\quad p_{z t}>$

Condition (17b) shows that blocking is the more likely the smaller are $Q, \lambda_{2}, \gamma$, and $\alpha$ and the larger is $\lambda_{1}$. The intuition for these findings is as follows. First, there is an opportunity cost of blocking. This cost is the larger the faster is technological progress in sector 2 , that is, the larger is $\gamma$. Second, blocking new technologies increases the relative price of $z$, benefits the skilled agents as producers of $\mathrm{z}$ but hurts them consumers of $\mathrm{z}$. The former effect is the stronger relative to the latter the more protected is sector $z$ from world market competition (i.e. the smaller is $Q$ ) and the larger is the income transfer from the unskilled to the skilled BBR, Braz. Bus. Rev. (Eng. ed., Online),

www.bbronline.com.br 
(i.e. the larger $\lambda_{1} \lambda_{2}$, the mass of the unskilled relative to the skilled, and the larger $1-\alpha$, the expenditure share of $z$ ).

\section{CONCLUSIONS}

In this section we summarize and compare the results obtained above. First, with respect to the adoption of new technologies our model indicates that under free trade or a tariff there is no resistance. The best technology available will always be used. On the other had, with a quota the groups with power to resist will stop the adoption of new technologies. Therefore, in our model, there is no equivalence between a tariff and a quota. The reason for this is the following. The utility of a skilled worker increases with his income that is equal to the marginal productivity of a type 2 labor times the relative price of the good that they produced $(\operatorname{good} z)$. Under free trade or a tariff the relative prices are given and adoption of technology does not affect the relative price of the goods produced in the economy. Therefore, to maximize their income (and their utility) the skilled workers just have to increased their productivity as much as possible. That is, they have to allow the use of the most productive technology. On the other hand, with a quota, the blocking of new technologies increases the relative price of the good intensive in skilled labor. Therefore, there are conditions under which, even though the skilled workers have smaller productivity, they have higher income. That is, the increment of the relative price is more than sufficient to compensate the reduction in the productivity, increasing the income of the skilled workers.

Two other results come as a consequence of this behavior toward the adoption of technology. First, even controlling for skill level (or human capital), on average, the workers in the economy with quota will have smaller productivity than the workers in the economy with free trade or with tariff. Second, the income per capita in the economy with quota is smaller than the income per capita in the economy with free trade or with a tariff. The reason is that under a quota the workers in the sector intensive in skill labor will not be using the most productive technology. Therefore, they will display smaller productivity and the whole economy will have a smaller income per capita.

Finally, we should also notice the effects of trade policies on income distribution. That is, even though the workers in the economy have smaller productivity and the income per capita is smaller, the skilled workers are better off under the quota arrangement. The reason is that the income of the skilled workers is higher with a quota than with free trade or a tariff. In other words, they have a bigger part in a smaller pie.

For future research we should introduce capital in the model and allow its free mobility across countries. The introduction of capital would allow the use of a more general production function. Besides, the introduction of capital would generate another constraint in the decision to block new technologies. Less productive technologies would affect the productivity of capital and thus investment. That is, resistance to new technologies would reduce investment, the capital stock and the capital-labor ratio affecting the productivity and the income of the skilled workers. 


\section{APPENDIX}

Proof of Lemma 1. Following the four steps outlined in the main text it is straightforward to derive the equilibria. The results are as follows. If $s=(a, t, t) \in S$,

then:

$$
\begin{aligned}
& \hat{p}(s)=\left\{1, \eta \pi^{t} \gamma^{-t}, \pi^{t}, \eta \pi^{t} \gamma^{-1}, \eta \pi^{t}\right\}, \\
& \hat{x}_{1}(s)=\left\{\alpha \pi^{t},(1-\alpha) \gamma^{t} \eta^{-1}, 1,0,0\right\}, \\
& \hat{x}_{2}(s)=\left\{\alpha \eta \pi^{t},(1-\alpha) \gamma^{t}, 0,0,1\right\}, \\
& \hat{x}_{3}(s)=\left\{\lambda_{1} \pi^{t}, 0, \lambda_{1}, 0,0\right\}, \\
& \hat{x}_{4}(s)=\left\{0, \lambda_{2} \gamma^{t}, 0,0, \lambda_{2}\right\}, \\
& \hat{x}_{5}(s)=\left\{-\lambda_{1}(1-\alpha) \pi^{t}+\lambda_{2} \alpha \eta \pi^{t}, \lambda_{1}(1-\alpha) \gamma^{t} \eta^{-1}-\lambda_{2} \alpha \gamma^{t}, 0,0,0\right\} .
\end{aligned}
$$

If $s=(a, b, t) \in S$ in $\mathrm{S}$ with $b<t$, then

$$
\begin{aligned}
& \hat{p}(s)=\left\{1, \eta \pi^{t} \gamma^{-t}, \pi^{t}, \eta \pi^{t} \gamma^{a-t}, \eta \pi^{t} \gamma^{b-t}\right\}, \\
& \hat{x}_{1}(s)=\left\{\alpha \pi^{t},(1-\alpha) \gamma^{t} \eta^{-1}, 1,0,0\right\}, \\
& \hat{x}_{2}(s)=\left\{\alpha \pi^{t},(1-\alpha) \gamma^{t} \eta^{-1}, 1,0,0\right\}, \\
& \hat{x}_{3}(s)=\left\{\pi^{t}\left(\lambda_{1}+\lambda_{2}\right), 0, \lambda_{1}+\lambda_{2}, 0,0\right\}, \\
& \hat{x}_{4}(s)=\{0,0,0,0,0\}, \\
& \hat{x}_{5}(s)=\left\{-\left(\lambda_{1}+\lambda_{2}\right)(1-\alpha) \pi^{t},\left(\lambda_{1}+\lambda_{2}\right)(1-\alpha) \gamma^{t} \eta^{-1}, 0,0,0\right\} .
\end{aligned}
$$

\section{- Proof}

of Proposition 1. We start with the second-period problem, (5b). Using (18a) and (18b) we obtain the indirect utilities:

$$
\begin{aligned}
& u(a, 2,2)=\frac{\left(\alpha \eta \pi^{2}\right)^{\alpha \rho}\left[(1-\alpha) \gamma^{2}\right]^{(1-\alpha) \rho}}{\rho} \\
& u(-1,0,2)=u(0,1,2)=\frac{\left(\alpha \eta \pi^{2}\right)^{\alpha \rho}\left[(1-\alpha) \gamma^{2}\right]^{(1-\alpha) \rho}}{\rho \eta^{\rho}} .
\end{aligned}
$$

Since $\eta>1, b=2$ is the optimal choice. This immediately implies the continuation utility:

$$
v(a, b, 1)=\frac{\left(\alpha \eta \pi^{2}\right)^{\alpha \rho}\left[(1-\alpha) \gamma^{2}\right]^{(1-\alpha) \rho}}{\rho} .
$$

Note that this shows that the continuation utility is independent of the realization of $(a, b, 1)$. Using this fact, the first-period problem, (5a), is equivalent to maximizing the period utility in the first period. It is given by

$$
\begin{aligned}
& u(0,1,1)=\frac{(\alpha \eta \pi)^{\alpha \rho}[(1-\alpha) \gamma]^{(1-\alpha) \rho}}{\rho} \\
& u(-1,0,1)=\frac{(\alpha \eta \pi)^{\alpha \rho}[(1-\alpha) \gamma]^{(1-\alpha) \rho}}{\rho \eta^{\rho}} .
\end{aligned}
$$


Again since $\eta>1$ the optimal choice is $b=1$. Given that the initial state is $(-1,0,0)$, using $b=$ $t$ and the law of motion implies that $s=(t-1, t, t)$ in both periods. This completes the proof.

Proof of Lemma 3. The derivation of the different equilibria is straightforward once it is clarified where the two types work. We will only sketch the key arguments.

Solving the firms' problems shows that the different wages (expressed in units of the numeraire $y$ ) are:

$$
w_{y t}=\pi^{t}, \quad w_{1 t}=(1+\tau) \eta \pi^{t} \gamma^{a-t}, \quad w_{2 t}=(1+\tau) \eta \pi^{t} \gamma^{b-t} .
$$

The five cases of the lemma come about as follows.

First, if and only if $w_{1 t}<w_{2 t}<w_{\mathrm{y} t}$, then both types work in sector 1 . Specifically:

$$
\begin{aligned}
& \hat{p}(s)=\left\{1,(1+\tau) \eta \pi^{t} \gamma^{-t}, \pi^{t},(1+\tau) \eta \pi^{t} \gamma^{a-t},(1+\tau) \eta \pi^{t} \gamma^{b-t}\right\}, \\
& \hat{x}_{1}(s)=\left\{\alpha \pi^{t},(1-\alpha) \gamma^{t}[(1+\tau) \eta]^{-1}, 1,0,0\right\}, \\
& \hat{x}_{2}(s)=\left\{\alpha \pi^{t},(1-\alpha) \gamma^{t}[(1+\tau) \eta]^{-1}, 1,0,0\right\}, \\
& \hat{x}_{3}(s)=\left\{\left(\lambda_{1}+\lambda_{2}\right) \pi^{t}, 0, \lambda_{1}+\lambda_{2}, 0,0\right\}, \\
& \hat{x}_{4}(s)=\{0,0,0,0,0\}, \\
& \hat{x}_{5}(s)=\left\{-\left(\lambda_{1}+\lambda_{2}\right)(1-\alpha) \pi^{t},\left(\lambda_{1}+\lambda_{2}\right)(1-\alpha) \gamma^{t}[(1+\tau) \eta] b^{-1}, 0,0,0\right\} .
\end{aligned}
$$

Since then $z$ is not produced it must be imported and there is no additional restriction. This is case (i).

Second, if and only if $w_{1 t}<w_{2 t}=w_{y t}$, then type 1 works in sector 1 and type 2 is indifferent between working in either sector. Specifically:

$$
\begin{aligned}
& \hat{p}(s)=\left\{1,(1+\tau) \eta \pi^{t} \gamma^{-t}, \pi^{t},(1+\tau) \eta \pi^{t} \gamma^{a-t},(1+\tau) \eta \pi^{t} \gamma^{b-t}\right\}, \\
& \hat{x}_{1}(s)=\left\{\alpha \pi^{t},(1-\alpha) \gamma^{t}[(1+\tau) \eta]^{-1}, 1,0,0\right\}, \\
& \hat{x}_{2}(s)=\left\{\alpha(1+\tau) \eta \pi^{t} \gamma^{b-t},(1-\alpha) \gamma^{b}, 0,0,1\right\}, \\
& \hat{x}_{3}(s)=\left\{\lambda_{1} \pi^{t}, 0, \lambda_{1}, 0,0\right\}, \\
& \hat{x}_{4}(s)=\left\{0, \lambda_{2} \gamma^{b}, 0,0, \lambda_{2}\right\}, \\
& \hat{x}_{5}(s)=\left\{-\lambda_{1}(1-\alpha) \pi^{t}+\lambda_{2} \alpha(1+\tau) \eta \pi^{t} \gamma^{b-t}, \lambda_{1}(1-\alpha) \gamma^{t}[(1+\tau) \eta]^{-1}-\lambda_{2} \alpha \gamma^{b}, 0,0,0\right\} .
\end{aligned}
$$

The additional requirement that $z$ be imported leads to the restriction $l>\alpha-\lambda_{1} \lambda_{2}^{-1}(1-\alpha)$. Given that (7) holds, the right-hand side is negative, so $z$ is imported for all $l \in[0,1]$. This is case (ii). 
Third, if and only if $\mathrm{w}_{1 \mathrm{t}}<w_{y t}<w_{2 t}$ type 1 works in sector 1 and type 2 works in sector 2. Specifically:

$$
\begin{aligned}
& \hat{p}(s)=\left\{1,(1+\tau) \eta \pi^{t} \gamma^{-t}, \pi^{t},(1+\tau) \eta \pi^{t} \gamma^{a-t},(1+\tau) \eta \pi^{t} \gamma^{b-t}\right\}, \\
& \hat{x}_{1}(s)=\left\{\alpha \pi^{t},(1-\alpha) \gamma^{t}[(1+\tau) \eta]^{-1}, 1,0,0\right\}, \\
& \hat{x}_{2}(s)=\left\{\alpha(1+\tau) \eta \pi^{t} \gamma^{b-t},(1-\alpha) \gamma^{b}, 0,0,1\right\}, \\
& \hat{x}_{3}(s)=\left\{\lambda_{1} \pi^{t}, 0, \lambda_{1}, 0,0\right\}, \\
& \hat{x}_{4}(s)=\left\{0, \lambda_{2} \gamma^{b}, 0,0, \lambda_{2}\right\}, \\
& \hat{x}_{5}(s)=\left\{-\lambda_{1}(1-\alpha) \pi^{t}+\lambda_{2} \alpha(1+\tau) \eta \pi^{t} \gamma^{b-t}, \lambda_{1}(1-\alpha) \gamma^{t}[(1+\tau) \eta]^{-1}-\lambda_{2} \alpha \gamma^{b}, 0,0,0\right\} .
\end{aligned}
$$

The additional restriction that $\mathrm{z}$ be imported leads to

$$
\imath>\alpha+\lambda_{1}^{-1} \lambda_{2}(1-) \alpha \gamma^{b-a} .
$$

This is case (iii).

Fourth, if and only if $w_{y t}=w_{1 t}<w_{2 t}$, then type 2 works in sector 2 and type 1 is indifferent between working in either sector. Specifically:

$$
\begin{aligned}
& \hat{p}(s)=\left\{1, \pi^{t} \gamma^{-a}, \pi^{t}, \pi^{t}, \pi^{t} \gamma^{b-a}\right\}, \\
& \hat{x}_{1}(s)=\left\{\alpha \pi^{t},(1-\alpha) \gamma^{a}, l, 1-l, 0\right\}, \quad l \in\left(\alpha+\lambda_{1}^{-1} \lambda_{2} \alpha \gamma^{b-a}, 1\right], \\
& \hat{x}_{2}(s)=\left\{\alpha \pi^{t} \gamma^{b-a},(1-\alpha) \gamma^{b}, 0,0,1\right\}, \\
& \hat{x}_{3}(s)=\left\{\lambda_{1} l \pi^{t}, 0, \lambda_{1} l, 0,0\right\}, \\
& \hat{x}_{4}(s)=\left\{0, \lambda_{1}(1-l) \gamma^{a}+\lambda_{2} \gamma^{b}, \lambda_{1}(1-\imath), 0, \lambda_{2}\right\}, \\
& \hat{x}_{5}(s)=\left\{\lambda_{1}(\alpha-\imath) \pi^{t}+\lambda_{2} \alpha \pi^{t} \gamma^{b-a}, \lambda_{1}(l-\alpha) \gamma^{a}-\lambda_{2} \alpha \gamma^{b}, 0,0,0\right\} .
\end{aligned}
$$

The additional requirement that $\mathrm{z}$ be imported leads to

$$
\iota<\alpha+\lambda_{1}^{-1} \lambda_{2}(1-) \alpha \gamma^{b-a}
$$

Given that (7), the right-hand side is smaller than 1 , so $l \in\left(\alpha+\lambda_{1}{ }^{-1} \lambda_{2}(1-) \alpha \gamma^{b-a}, 1\right]$. This is case (iv). Fifth, in all other cases, $z$ is exported because there is too little production of $y$ to export it. The conditions in (13) follow by logically negating (11) and (12). This is case (v).

Proof of Proposition 2. We start the proof by noting that under condition (14c) there are no period equilibria in which $\mathrm{z}$ is imported, so a recursive equilibrium cannot exist either. In contrast, under conditions (14a) and (14b) there are unique period equilibria in which $\mathrm{z}$ is imported. We study these two cases subsequently.

If (14a) holds, then we can restrict attention on the cases (i)-(iii) of Lemma 3, in which period equilibria with the desired properties exist. If $b-t=0$, then case (iii) applies. If $b-t<$ 0 , then cases (i) or (ii) apply. Note that which one of the two latter is relevant does not matter for the current purpose because (10) implies that the indirect period utility in both cases is found to be equal.

Using (20)-(22), we obtain the indirect utilities: 


$$
\begin{aligned}
& u(a, 2,2)=\frac{\left[\alpha(1+\tau) \eta \pi^{2}\right]^{\alpha \rho}\left[(1-\alpha) \gamma^{2}\right]^{(1-\alpha) \rho}}{\rho} \\
& u(-1,0,2)=u(0,1,2)=\frac{\left[\alpha(1+\tau) \eta \pi^{2}\right]^{\alpha \rho}\left[(1-\alpha) \gamma^{2}\right]^{(1-\alpha) \rho}}{\rho[(1+\tau) \eta]^{\rho}}
\end{aligned}
$$

Since $(1+\tau) \eta>1, b=2$ is the optimal choice. This immediately implies the continuation utility:

$$
v(a, b, 1)=\frac{\left[\alpha(1+\tau) \eta \pi^{2}\right]^{\alpha \rho}\left[(1-\alpha) \gamma^{2}\right]^{(1-\alpha) \rho}}{\rho} .
$$

Note that this shows that the continuation utility is independent of the realization of $(a, b, 1)$. Using this fact, the first-period problem, (5a), is equivalent to maximizing the period utility in the first period, which is given by:

$$
\begin{aligned}
& u(0,1,1)=\frac{[\alpha(1+\tau) \eta \pi]^{\alpha \rho}[(1-\alpha) \gamma]^{(1-\alpha) \rho}}{\rho}, \\
& u(-1,0,1)=\frac{[\alpha(1+\tau) \eta \pi]^{\alpha \rho}[(1-\alpha) \gamma]^{(1-\alpha) \rho}}{\rho[(1+\tau) \eta]^{\rho}} .
\end{aligned}
$$

Thus, the optimal choice is $b=1$. Given that the initial state is $(-1,0,0)$, using $b=t$ and the law of motion implies that $s=(t-1, t, t)$ in both periods.

If (14b) holds, then cases (i)-(ii) or (iv) of Lemma 3 are relevant. The latter applies if $b-t=$ 0 whereas the former apply if $b-1<0$. The steps of the proof are now exactly the same as in the previous case. They are left as an exercise for the reader. This completes the proof.

Proof of Lemma 4. We start with the proof of case (i). Solving the problems of the two representative firms one finds $w_{y t}=\pi^{t}, w_{1 t}=\gamma^{a} \mathrm{p}_{\mathrm{zt}}, w_{2 t}=\gamma^{b} p_{z t}$. Since requiring type 1 to work in both sector and type 2 to work in sector 2 is equivalent to $w_{y t}=w_{1 t}<w_{2 \mathrm{i}}$, we have

$$
p_{z t}=\pi^{t} \gamma^{-a}, \quad w_{y t}=w_{1 t} \pi^{t}, \quad w_{2 t}=\pi^{t} \gamma^{b-a} .
$$

Solving the problems of the two representative agents, we find that the period expenditure shares of the two goods are $\alpha$ and $1-\alpha$. Using that $p_{z t}=\pi^{t} \gamma^{-a}$, the equilibrium quantities consumed by the two types therefore are:

$$
y_{1 t}=\alpha \pi^{t}, \quad z_{1 t}=(1-\alpha) \gamma^{a}, \quad y_{2 t}=\alpha \pi^{t} \gamma^{b-a}, \quad z_{2 t}=(1-\alpha) \gamma^{b} .
$$

The period quantities of the two goods that are produced and their period exports and imports can be determined as follows. Using that the sum of the period quantities of $\mathrm{z}$ consumed by both types must equal the period production of $\mathrm{z}$ plus the period imports of $\mathrm{z}$ we get:

$$
\lambda_{1} z_{2 t}+\lambda_{2} z_{2 t}=\left(\lambda_{1} l_{2 t} \gamma^{a}+\lambda_{2} \gamma^{b}\right)(1+Q) \text {. }
$$

Substituting $\mathrm{z}_{\mathrm{it}}$ from above into this expression and solving for $\mathrm{l}_{1 \mathrm{t}}$ gives:

$$
l_{2 t}=\frac{(1-\alpha)-\lambda_{1}^{-1} \lambda_{2}(\alpha+Q) \gamma^{b-a}}{1+Q} .
$$

Using $1_{1 \mathrm{t}}=1-1_{2 \mathrm{t}}$ it is now straightforward to compute the remaining variables. 
In sum, in case (i) the equilibrium is given by

$$
\begin{aligned}
\hat{p}(s)= & \left\{1, \pi^{t} \gamma^{-a}, \pi^{t}, \pi^{t}, \pi^{t} \gamma^{b-a}\right\}, \\
\hat{x}_{1}(s)= & \left\{\alpha \pi^{t},(1-\alpha) \gamma^{a},\left[1+\lambda_{1}^{-1} \lambda_{2}(\alpha+Q) \gamma^{b-a}\right](1+Q)^{-1},\right. \\
& {\left.\left[(1-\alpha)-\lambda_{1}^{-1} \lambda_{2}(\alpha+Q) \gamma^{b-a}\right](1+Q)^{-1}, 0\right\}, } \\
\hat{x}_{2}(s)= & \left\{\alpha \pi^{t} \gamma^{b-a},(1-\alpha) \gamma^{b}, 0,0,1\right\}, \\
\hat{x}_{3}(s)= & \left\{\left(\lambda_{1}+\lambda_{2} \gamma^{b-a}\right) \pi^{t}(\alpha+Q)(1+Q)^{-1}, 0,\left(1+\lambda^{-1} \lambda_{2} \gamma^{b-a}\right)(\alpha+Q)(1+Q)^{-1}, 0,0\right\}, \\
\hat{x}_{4}(s)= & \left\{0,\left(\lambda_{1} \gamma^{a}+\lambda_{2} \gamma^{b}\right)(1-\alpha)(1+Q)^{-1}, 0,\left[(1-\alpha)-\lambda_{1}^{-1} \lambda_{2}(\alpha+Q) \gamma^{b-a}\right](1+Q)^{-1}, \lambda_{2}\right\}, \\
\hat{x}_{5}(s)= & \left\{\left(\lambda_{1}+\lambda_{2} \gamma^{b-a}\right)(1-\alpha) \pi^{t} Q(1+Q)^{-1},\left(\lambda_{1} \gamma^{a}+\lambda_{2} \gamma^{b}\right)(1-\alpha) Q(1+Q)^{-1}, 0,0,0\right\} .
\end{aligned}
$$

The final order of business is to check that $p_{z t}>p_{z t}{ }^{*}$, that the quota is binding, and that $1_{z 1 t} \in(0$, 1). The first requirement is implied by $\eta<\gamma$ and $\mathrm{a} \leq t-1$ :

$$
p_{z t}^{*}=\eta \frac{\pi^{t}}{\gamma^{t}}=\frac{\eta}{\gamma} \frac{\pi^{t}}{\gamma^{t-1}}<\frac{\pi^{t}}{\gamma^{t-1}} \leq \frac{\pi^{t}}{\gamma^{a}}=p_{z t} .
$$

The second requirement can be checked as follows. If $b<t$, then under the quota $z_{t}{ }^{*}=(\lambda$ $\left.{ }_{1} \gamma^{a}+\lambda_{2} \gamma^{b}\right)(1-\alpha) Q(1+Q)^{-1}$ and under free trade $z_{t}{ }^{*}\left(\lambda_{1}+\lambda_{2}\right)(1-\alpha) \gamma^{t} \eta^{-1}$; see Lemma 1 for the latter. Thus the quota binds:

$$
\frac{\left(\lambda_{1} \gamma^{a}+\lambda_{2} \gamma^{b}\right)(1-\alpha) Q}{(1+Q)}<\frac{\left(\lambda_{1} \gamma^{a}+\lambda_{2} \gamma^{b}\right) \gamma(1-\alpha)}{\eta} \leq \frac{\left(\lambda_{1}+\lambda_{2}\right)(1-\alpha) \gamma^{t}}{\eta} .
$$

If $b=t$, then from Lemma 1 we can see that under free trade $z_{t}{ }^{*} \lambda_{1}(1-\alpha) \gamma^{t} \eta^{-1}-\lambda_{2} \alpha \gamma^{t}$. Using the right inequality of condition (16b), we now get:

$$
Q<\frac{\lambda_{1}(1-\alpha)}{\lambda_{2} \gamma}-\alpha<\frac{\lambda_{1}(1-\alpha)}{\lambda_{2} \eta}-\alpha<\frac{\lambda_{1}(1-\alpha)}{\lambda_{2} \eta}-\alpha+\frac{\lambda_{1}(1-\alpha) Q}{\lambda_{2}}\left[\frac{1}{\eta}-\frac{1}{\gamma}\right],
$$

which implies that the quota binds. The first part of the third requirement, $1_{z 1 t}>0$, it trivially satisfied. The second part, $1_{z 1 t}<1$, is equivalent to (16a).

We now turn to case (ii). We will only highlight the differences to the proof of case (i) and leave the rest as an exercise to the reader. We know that $1_{1 t}=0$ and $l_{2 t}=1$ because type $\mathrm{i}$ only works in sector $i$. Thus it must be that $w_{1 t} \leq w_{y t}<w_{2 t}$, implying that we cannot derive $p_{z t}$ immediately from the equality of two wages. ${ }^{12} p_{z t}$ can be obtained by using that the sum of the period quantities of $z$ consumed of both types must equal period production of $z$ plus the period imports of $\mathrm{z}$. This gives:

$$
p_{z t}=\frac{\lambda_{1}(1-\alpha) \pi^{t}}{\lambda_{2}(\alpha+Q) \gamma^{b}}
$$

\footnotetext{
${ }^{12}$ Note that $\mathrm{w}_{1 \mathrm{t}}=\mathrm{w}_{\mathrm{yt}}$ only for the parameter constellation, in which type 1 is indifferent between working in either sector but works in sector 1 only.
} 
Given $p_{z t}$, it is straightforward to derive the other variables:

$$
\begin{aligned}
\hat{p}(s)= & \left\{1, \lambda_{1} \lambda_{2}^{-1}(1-\alpha) \pi^{t}(\alpha+Q)^{-1} \gamma^{-b}, \pi^{t}, \lambda_{1} \lambda_{2}^{-1}(1-\alpha) \pi^{t}(\alpha+Q)^{-1} \gamma^{a-b},\right. \\
& \left.\lambda_{1} \lambda_{2}^{-1}(1-\alpha) \pi^{t}(\alpha+Q)^{-1}\right\}, \\
\hat{x}_{1}(s)= & \left\{\alpha \pi^{t}, \lambda_{1}^{-1} \lambda_{2}(\alpha+Q) \gamma^{b}, 1,0,0\right\}, \\
\hat{x}_{2}(s)= & \left\{\lambda_{1} \lambda_{2}^{-1} \alpha(1-\alpha) \pi^{t}(\alpha+Q)^{-1},(1-\alpha) \gamma^{b}, 0,0,1\right\}, \\
\hat{x}_{3}(s)= & \left\{\lambda_{1} \pi^{t}, 0, \lambda_{1}, 0,0\right\}, \\
\hat{x}_{4}(s)= & \left\{0, \lambda_{2} \gamma^{b}, 0,0, \lambda_{2}\right\}, \\
\hat{x}_{5}(s)= & \left\{\lambda_{1}(1-\alpha) \pi^{t} Q(\alpha+Q)^{-1}, \lambda_{2} Q \gamma^{b}, 0,0,0\right\} .
\end{aligned}
$$

We complete case (ii) by showing that $p_{z t}>p_{z t}{ }^{*}$, that the quota is binding, and that $w_{1 t} \leq w_{y t}$ $<w_{2 t}$. The first requirement is equivalent to:

$$
Q<\frac{\lambda_{1}(1-\alpha) \gamma^{t-b}}{\lambda_{2} \eta}-\alpha,
$$

which is implied by (16b). The second requirement can be checked as follows. If $b<t$, then the imports under the quota are $\lambda_{2} Q \gamma^{b}$ and the imports under free trade are $\left(\lambda_{1}+\lambda_{2}\right)(1-$ $\alpha) \gamma^{t} \eta^{-1}$; see Lemma 1 for the latter. The right inequality of condition (16b) then implies that the quota binds:

$$
Q<\frac{\lambda_{1}(1-\alpha)}{\lambda_{2}}-\alpha \leq \frac{\lambda_{1}(1-\alpha) \gamma^{t-b}}{\lambda_{2}}+(1-\alpha) \gamma^{t-b}
$$

If $b=t$, then the imports under free trade become $\lambda_{1}(1-\alpha) \gamma^{t} \eta^{-1}-\lambda_{2} \alpha \gamma^{t}$. Therefore, the right inequality of condition (16b) implies immediately that the quota binds. The third requirement is equivalent to

$$
\frac{\lambda_{1}(1-\alpha)}{\lambda_{2} \gamma^{b-a}}-\alpha \leq Q \leq \frac{\lambda_{1}(1-\alpha)}{\lambda_{2}}-\alpha,
$$

which is implied by (16b). Note that if there are equality signs in these two inequalities, then still type 1 works in the y sector and type 2 in sector 2 . This can be seen as follows. If $Q=\frac{\lambda_{1}(1-\alpha)}{\lambda_{2} \gamma^{b-a}}-\alpha$, then $1_{z 1}=0$ in case (i), that is, the first type no longer works in sector 2. If $\mathrm{Q}=$ $Q=\frac{\lambda_{1}(1-\alpha)}{\lambda_{2}}-\alpha^{-} \alpha$, then $l_{y 1}=0$ in case (iii), that is, the second type no longer works in the $y$ sector.

We continue with case (iii). Given that type 2 works in both sectors $w_{1 t}<w_{y t}=w_{2 t}$, implying that $p_{z t}=\pi^{t} \gamma^{b}$. The rest of the equilibrium can be computed in much the same way as the equilibrium in case (i). The result is: 


$$
\begin{aligned}
& \hat{p}(s)=\left\{1, \pi^{t} \gamma^{-b}, \pi^{t}, \pi^{t} \gamma^{-a}, \pi^{t}\right\}, \\
& \hat{x}_{1}(s)=\left\{\alpha \pi^{t},(1-\alpha) \gamma^{b}, 1,0,0\right\}, \\
& \hat{x}_{2}(s)=\left\{\alpha \pi^{t},(1-\alpha) \gamma^{b},\left[(\alpha+Q)-\lambda_{1} \lambda_{2}^{-1}(1-\alpha)\right](1+Q)^{-1}, 0,\left(\lambda_{1} \lambda_{2}^{-1}+1\right)(1-\alpha)(1+Q)^{-1}\right\}, \\
& \hat{x}_{3}(s)=\left\{\left(\lambda_{1}+\lambda_{2}\right)(\alpha+Q) \pi^{t}(1+Q)^{-1}, 0,\left(\lambda_{1} \lambda_{2}^{-1}+1\right)(\alpha+Q)(1+Q)^{-1}, 0,0\right\}, \\
& \hat{x}_{4}(s)=\left\{0,\left(\lambda_{1}+\lambda_{2}\right) \gamma^{b}(1-\alpha)(1+Q)^{-1}, 0,0,\left(\lambda_{1} \lambda_{2}^{-1}+1\right)(1-\alpha)(1+Q)^{-1}\right\}, \\
& \hat{x}_{5}(s)=\left\{-\left(\lambda_{1}+\lambda_{2}\right) \pi^{t}(1-\alpha) Q(1+Q)^{-1},\left(\lambda_{1}+\lambda_{2}\right) \gamma^{b}(1-\alpha) Q(1+Q)^{-1}, 0,0,0\right\} .
\end{aligned}
$$

Checking the three requirements in this case is straightforward. First, the quota can be shown to bind without further restrictions. Second, $p_{z t}>p_{z t}{ }^{*}$ is satisfied if and only if $b<t$. Third, $l_{z t}>0$ without further restrictions and $l_{z t}<1$ is equivalent to (16b).

At the end, we address case (iv). This amounts to showing that there cannot be other equilibria. The first possibility that we have not so far considered is that $w_{y}<w_{1}<w_{2}$ in equilibrium. This cannot be because all agents would work in the second sector and $z$ would be exported. The second possibility that we have not so far considered is that $w_{1}<w_{2}<w_{\mathrm{y}}$ in equilibrium. In this case, all agents would work in the first sector. If $b=t$, then the requirement that $w_{2}<w_{\mathrm{y}}$ implies that $p_{z}<p_{z}{ }^{*}$, which is not allowed. If $b<t$, then the unique equilibrium under free trade also has all agents working in the first sector. But that means that the quota must equal the imports under free trade, and so it is not binding. This completes the proof.

Proof of Proposition 3. In case (i) of the proposition, case (i) of Lemma 4 implies for all possible realizations of the state. We start with the second period. The state at the beginning of the second period can have two realizations only: $(-1,0,1)$ or $(0,1,1)$. The former (latter) obtains if the new technology was blocked (adopted) in the first period. If the state is $(-1,0$, 1), then the second-period utility follows from (23) of Lemma 4:

$$
\begin{aligned}
& u(0,2,2)=\frac{\left(\alpha \pi^{2}\right)^{\alpha \rho}(1-\alpha)^{(1-\alpha) \rho} \gamma^{2 \rho}}{\rho}, \\
& u(0,1,2)=\frac{\left(\alpha \pi^{2}\right)^{\alpha \rho}(1-\alpha)^{(1-\alpha) \rho} \gamma^{\rho}}{\rho} .
\end{aligned}
$$

Thus, $b=2$ is the optimal choice. If the state is $(0,1,1)$, then the second-period utility is either

$$
u(1,2,2)=\frac{\left(\alpha \pi^{2}\right)^{\alpha \rho}(1-\alpha)^{(1-\alpha) \rho} \gamma^{(2-\alpha) \rho}}{\rho}
$$

or $u(0,1,2)$ as computed above. Again, $b=2$ is the optimal choice. Using these two results, the continuation utility can be computed:

$$
\begin{aligned}
& v(-1,0,1)=\frac{\left(\alpha \pi^{2}\right)^{\alpha \rho}(1-\alpha)^{(1-\alpha) \rho} \gamma^{2 \rho}}{\rho}, \\
& v(0,1,1)=\frac{\left(\alpha \pi^{2}\right)^{\alpha \rho}(1-\alpha)^{(1-\alpha) \rho} \gamma^{(2-\alpha) \rho}}{\rho} .
\end{aligned}
$$


We now turn to the first period. Recall that at the beginning of the first period the state is $(-1$, $0,0)$. If the new technology is adopted, then the previous expressions imply the following present value of utility:

$$
u(0,1,1)+\beta v(0,1,1)=\frac{(\alpha \pi)^{\alpha \rho}(1-\alpha)^{(1-\alpha) \rho} \gamma^{\rho}}{\rho}+\beta \frac{\left(\alpha \pi^{2}\right)^{\alpha \rho}(1-\alpha)^{(1-\alpha) \rho} \gamma^{(2-\alpha) \rho}}{\rho} .
$$

If the new technology is blocked, then the present value of utility is

$$
u(-1,0,1)+\beta v(-1,0,1)=\frac{(\alpha \pi)^{\alpha \rho}(1-\alpha)^{(1-\alpha) \rho} \gamma^{\alpha \rho}}{\rho}+\beta \frac{\left(\alpha \pi^{2}\right)^{\alpha \rho}(1-\alpha)^{(1-\alpha) \rho} \gamma^{2 \rho}}{\rho} .
$$

Comparing the last two expressions shows that blocking is optimal if and only if (17b) in the text holds.

In case (ii) of the proposition, case (i) of Lemma 4 applies if $(b-a)=1$ and case (ii) applies if $(b-a)=2$. We again start with the second period. If the state is $(-1,0,1)$, then the secondperiod utility $u(0,1,2)$ is as before and $u(0,2,2)$ can be computed by using (24) of Lemma 4:

$$
u(0,2,2)=\frac{\left[\alpha \pi^{2} \lambda_{1}(1-\alpha)\right]^{\alpha \rho}(1-\alpha)^{(1-\alpha) \rho} \gamma^{2(1-\alpha) \rho}}{\rho\left[\lambda_{2}(\alpha+Q)\right]^{\alpha \rho}} .
$$

Thus, $\mathrm{b}=2$ is the optimal choice. If the state is $(0,1,1)$, then the second-period utilities $u(1$, $2,2)$ and $u(0,1,2)$ are as in the previous case and again $b=2$ is the optimal choice. Using these two results, the continuation utility can be computed:

$$
\begin{aligned}
& v(-1,0,1)=\frac{\left[\alpha \pi^{2} \lambda_{1}(1-\alpha)\right]^{\alpha \rho}(1-\alpha)^{(1-\alpha) \rho} \gamma^{2(1-\alpha) \rho}}{\rho\left[\lambda_{2}(\alpha+Q)\right]^{\alpha \rho}}, \\
& v(0,1,1)=\frac{\left(\alpha \pi^{2}\right)^{\alpha \rho}(1-\alpha)^{(1-\alpha) \rho} \gamma^{(2-\alpha) \rho}}{\rho} .
\end{aligned}
$$

We now turn to the first period. Recall that at the beginning of the first period the state is $(-1$, $0,0)$. If the new technology is adopted, then the present value of utility, $u(0,1,1)+\beta v(0,1$, 1 ), is given by (26). If the new technology is blocked, then the present value of utility is

$$
u(-1,0,1)+\beta v(-1,0,1)=\frac{(\alpha \pi)^{\alpha \rho}(1-\alpha)^{(1-\alpha) \rho} \gamma^{\alpha \rho}}{\rho}+\beta \frac{\left[\alpha \pi^{2} \lambda_{1}(1-\alpha)\right]^{\alpha \rho}(1-\alpha)^{(1-\alpha) \rho} \gamma^{2(1-\alpha) \rho}}{\rho\left[\lambda_{2}(\alpha+Q)\right]^{\alpha \rho}} .
$$

Comparing the last two expressions shows that blocking is optimal if and only if (17d) in the text holds.

In case (iii) the proof is exactly the same as the proof of the existence and uniqueness of a recursive equilibrium under free trade or under a tariff. The key feature of the period equilibrium again is that $y_{2 t}$ and $z_{2 t}$ do not depend on a or $t-b$ but that $z_{2 t}$ increases in $b$. Consequently the indirect period utility of type 2 is independent of a and increases in $b$, implying that the best technology is adopted in all periods. The formal details of the proof are left as an exercise to the reader. 
In case (iv) the proof has two subcases. First, if

$$
Q>\frac{\lambda_{1}(1-\alpha)}{\lambda_{2}}-\alpha
$$

then case (iii) of Lemma 4 applies. So suppose that a recursive equilibrium exists. Since $y_{2 t}$ and $z_{2 t}$ do not depend on a or $t-b$ but $z_{2 t}$ increases in $b$, for the same reasons as in case (iii) it is optimal to adopt the best technology. However, a period equilibrium can exist only if $b<t$, so we have a contradiction. Second, if

$$
\frac{\lambda_{1}(1-\alpha)}{\lambda_{2} \eta}-\alpha \leq Q \leq \frac{\lambda_{1}(1-\alpha)}{\lambda_{2}}-\alpha,
$$

then case (ii) of Lemma 4 may apply if $b-t$ is chosen sufficiently large. So suppose that a recursive equilibrium exists. In the corresponding period equilibrium $y_{2 t}$ and $z_{2 t}$ again do not depend on a or $t-b$ but $z_{2 t}$ increases in $b$. For the same reasons as in case (iii) it is then optimal to adopt the best technology. Thus, $b-t=0$, which gives a contradiction. Filling in the formal details of the two proof in case (iv) is left as an exercise to the reader.

\section{REFERENCES}

[1] M Baily. Competition, regulation and efficiency in service industries. Brookings Papers on Economic Activity, Microeconomics:71-103, 1993.

[2] M Baily and $\mathrm{H}$ Gersbach. Efficiency in manufacturing and the need for global competition. Brookings Papers on Economic Activity, Microeconomics:307-347, 1995.

[3] Jagdish Bhagwati. On the equivalence of tariffs and quotas. In Richard Caves, editor, Trade, growth and balance of payments. Rand-McNally, 1965.

[4] Jagdish Bhagwati. More on the equivalence of tariffs and quotas. American Economic Review, pages 142-146, 1968.

[5] David Dollar and Edward N Wolff. Competitiveness, convergence, and international specialization. MIT Press, 1993.

[6] Pedro C Ferreira and Jose Luis Rossi. New evidence on trade liberalization and productivity growth. Working paper, Fundacao Getulio Vargas, 2001.

[7] James Harrigan. Cross-country comparisons of industry total factor productivity: theory and evidence. mimeo, Federal Reserve Bank of New York, 1996.

[8] James Harrigan. Estimation of cross-country differences in industry production functions. Working Paper 6121, NBER, 1997.

[9] T Holmes and J Schmitz. Resistance to new technology and trade between areas. Federal Reserve Bank of Minneapolis Quarterly Review, 19:2\{17, 1995.

[10] Euysung Kim. Trade liberalization and productivity growth in korean manufacturing industries. Journal of Development Economics, 62:55\{83, 2000.

[11] Joel Mokyr. The lever of riches: technological creativity and economic progress. Oxford University Press, 1990. 
[12] Joel Mokyr. Technological inertia in economic history. Journal of Economic History, 52:325\{38, June 1992.

[13] Joel Mokyr. Progress and inertia in technological change, chapter 8. The University of Chicago Press, 1994.

[14] Marc-Andreas Muendler. Trade, technology and productivity: a study of brazilian manufacturers, 1986-1998. mimeo, University of California, Berkeley, 2001.

[15] Mancur Olson. The rise and decline of nations. Yale University Press, 1982.

[16] S Parente and E Prescott. Monopoly rights: a barrier to riches. American EconomicReview, 89:1216\{33, 1999.

[17] Stephen Parente and Edward Prescott. Barriers to Riches. MIT Press, 2000.

[18] Nina Pavcnik. Trade liberalization, exit, and productivity improvements: evidence from chilean plants. Review of Economic Studies, 69:245 \{276, 2002.

[19] E Prescott. Needed: A theory of total factor productivity. International economic review, 39:525\{52, 1998.

[20] J Tybout, J de Mello, and V Corbo. The effects of trade reforms on scale and technical efficiency: new evidence from Chile. Journal of International Economics, 31:231\{250, 1991. 